\title{
A gestão de projetos como vantagem competitiva para internacionalização de empresas brasileiras
}

\author{
André Luiz Spinelli Schelini ${ }^{1}$, Cristina Dai Prá Martens e Marcos Roberto Piscopo
}

Universidade Nove de Julho - UNINOVE, São Paulo, SP, Brasil

\section{DETALHES DO ARTIGO}

\section{Histórico do artigo:}

Recebido: 16 de abril de 2017

Aceito: 26 de junho de 2017

Disponível online: 12 de dezembro de 2017

Sistema de Revisão "Double Blind Review"

Editor científico:

Ilan Avrichir

\section{Palavras-chaves:}

Maturidade em Gestão de Projetos Internacionalização de Empresas Visão Baseada em Recursos (RBV) Empresas Brasileiras

Vantagem Competitiva Sustentável.

\begin{abstract}
RESUMO
Este estudo concentra-se em três eixos teóricos: gestão de projetos (GP), internacionalização de empresas e visão baseada em recursos (RBV). Teve como objetivo analisar se, e de que forma, a maturidade em GP contribui para estratégia de atuação internacional de empresas brasileiras sob a perspectiva da RBV. Adotou-se estudo de múltiplos casos, de abordagem exploratória-descritiva, com análise intercaso e intracaso, em três empresas brasileiras. Como resultado inédito, o estudo identificou que cinco características da gestão madura de projetos: sustentabilidade do projeto, envolvimento dos stakeholders, definição de objetivos e estratégias, gerenciamento da mudança e gerenciamento de riscos, contribuem para a internacionalização das empresas pesquisadas, com implicação de vantagem competitiva sustentável, considerando a análise RBV-VRIO. A pesquisa apresenta contribuições para as empresas que estão ou pretendem iniciar sua internacionalização, de forma que obtenham melhores resultados utilizando a GP. Como uma limitação, aponta-se o número reduzido de casos investigados, o que pode também ser uma oportunidade para desenvolvimento de novos estudos.
\end{abstract}

(C) 2017 Internext | ESPM. All rights reserved!

\section{INTRODUÇÃO}

A evolução de uma empresa nacional a uma empresa internacional é um passo importante para empresas que desejam internacionalizar-se. Atualmente tem sido mais frequente o movimento de empresas nacionais considerarem a estratégia de internacionalização de suas operações como uma estratégia de sobrevivência do negócio (Penrose, 2006; Floriani \& Fleury, 2012). Clientes internacionais, global sourcing - cadeias de fornecimento global e joint ventures no exterior estão remodelando o ambiente corporativo das empresas. Essas rápidas mudanças exigem das empresas o desenvolvimento da competitividade em nível global (Cleland \& Ireland, 2008). Em um ambiente muito competitivo, há uma necessidade de identificar e compreender os fatores que impactam o desempenho internacional das empresas (Porter, 1996; Barney, 2001).
Nesse contexto, as empresas precisam buscar alternativas de gestão, estabelecer estruturas organizacionais diferenciadas e desenvolver novos recursos, com respostas para atender às demandas do mercado nacional e competir no mercado internacional (Shenhar \& Dvir, 2010). A gestão orientada para projetos apresenta-se como uma alternativa cada vez mais aceita pelas organizações para resolução dos desafios corporativos (Carvalho \& Rabechini, 2011; Kerzner, 2011). As empresas que adotaram a prática madura de gestão de projetos (GP) estão mais capacitadas ao sucesso na corrida pelo mercado (Kerzner, 2006). Os modelos de maturidade em GP permitem a realização de um processo de aprendizagem das práticas de GP adotadas pela organização e melhora das competências em GP (Carvalho \& Rabechini, 2011; Kerzner, 2006). 
A abertura de novos mercados fora do país de origem da empresa pode adotar diferentes modelos de negócios e estratégias (Root, 1987; Anderson \& Gatignon, 1986). Nesse sentido, a internacionalização torna-se uma decisão estratégica de expansão comercial (Porter, 1996; Hitt, Ireland \& Hoskisson, 2011; Penrose, 2006), que as empresas adotam de forma planejada. Porém, muitas empresas que buscam a internacionalização têm dificuldade de implementar modelos de gestão que contribuam de forma estratégica para esse fim (Hitt et al., 2011; Hollensen, 2011; Moreira \& Silveira, 2013). A GP é uma alternativa que pode contribuir nesse processo (Cleland \& Ireland, 2008).

Este estudo buscou analisar a implicação competitiva da prática madura da GP em empresas internacionais brasileiras, como premissa para expandir internacionalmente. Partiu-se do pressuposto que a gestão madura de projetos confere uma vantagem competitiva, e com isso a empresa pode ter um melhor desempenho em sua estratégia de internacionalização (Kerzner, 2006; Cleland \& Ireland, 2008; PMI, 2008). Para essa análise, optou-se por utilizar a perspectiva da Visão Baseada em Recursos - RBV (Wernerfelt, 1984; Barney, 1991; Peteraf, 1993; Penrose, 2006), que fornece insumos para analisar o comportamento estratégico internacional da empresa, concentrandose sobre o conjunto de recursos e competências únicas que pertencem ou que tenham sido adquiridos externamente pelas empresas (Barney, 1991; Penrose, 2006). As características dos recursos influenciam na capacidade da empresa de alcançar, ou não, uma determinada vantagem competitiva (Barney, 1991; Grant, 1991).

Os três eixos teóricos do trabalho (GP, internacionalização e RBV) possuem características que se assemelham em suas definições conceituais. O processo de internacionalização pode ser considerado um projeto pois está sujeito a metas fixas de tempo, custo e recursos, sendo realizado apenas uma vez e, portanto, é único. Ainda a internacionalização em sua totalidade é um objeto altamente complexo que necessita ser subdividido em unidades menores para tornar-se gerenciável como um todo, como subprojetos, e mesmo em etapas. Na perspectiva da RBV-VRIO, a maturidade em GP exige a excelência na prática de GP, fazendo com que a empresa adquira competências que passam a ser um recurso chave que contribui para o sucesso, sendo um recurso raro, de valor para o negócio, difícil de imitar, e a empresa se apropria com o tempo e o aprendizado gerado.

Assim, tem-se a seguinte pergunta de pesquisa: a maturidade em GP pode contribuir para a estratégia de internacionalização de empresas brasileiras? O objetivo deste artigo é analisar se, e de que forma, a maturidade em GP contribui para estratégia de atuação internacional de empresas brasileiras sob a perspectiva da RBV. Para seu desenvolvimento, adotou-se a metodologia de estudo de casos múltiplos com três casos de empresas que utilizam a gestão madura de projetos, e este recurso usado de forma estratégica para alcançar a internacionalização da empresa.

Como principais resultados, este estudo aponta que a maturidade em GP é uma vantagem competitiva da empresa e que este recurso contribui para estratégia de internacionalização de empresas brasileiras sob a perspectiva da RBV. Logo, os resultados deste estudo apresentam contribuições acadêmicas e práticas para o uso de métodos maduros da GP para a internacionalização das empresas brasileiras sob a ótica da RBV. Alguns limites do estudo devem ser considerados, a exemplo de limitada exploração de diferentes aspectos da GP nas empresas e do reduzido número de respondentes em cada caso.

Na sequência, o artigo retoma os temas centrais do estudo (seção 2), aborda o método de pesquisa (seção 3), apresenta os resultados e os analisa à luz da literatura (seção 4), e, por fim, faz as considerações finais (seção 5).

\section{REVISÃO DA LITERATURA}

\subsection{Gestão de Projetos e Maturidade}

Um projeto pode ser definido como um esforço temporário realizado seguindo um ciclo de vida específico para criar um único produto, serviço ou resultado através da mobilização de recursos humanos, materiais e financeiros (Muriithi \& Crawford, 2003; Stanleigh, 2007; PMI, 2008). A GP é o resultado de um conjunto de esforços organizados de forma estruturada e determinada, utilizando práticas e habilidades dentro de uma organização (Levine, 2002). Envolve o uso de metodologias, áreas de conhecimento, atividades interdependentes, e múltiplos recursos conduzidos pela tripla restrição (Webster \& Knutson, 2006), para cumprir requisitos

Internext | São Paulo, v.12, n. 3, p. 01-15, set./dez. 2017 
Tab. 1

Análise comparativa dos Modelos de Maturidade baseados no CMM.

\begin{tabular}{ccccccc}
\hline \multirow{2}{*}{ Nível } & \multirow{2}{*}{ CMM } & \multicolumn{5}{c}{ Modelos de Maturidade de Gerenciamento de Projetos } \\
\cline { 3 - 6 } & OPM3 & PMMM & KPMMM & MMGP & P3M3 \\
\hline 1 & Inicial & Padronização & Processos Iniciais & Linguagem Comum & Inicial & $\begin{array}{c}\text { Processo } \\
\text { Inicial }\end{array}$ \\
\hline 2 & Repetível & Medição & $\begin{array}{c}\text { Processos Estruturados e } \\
\text { Padronizados }\end{array}$ & Processos Comuns & Conhecido & $\begin{array}{c}\text { Processo } \\
\text { Repetível }\end{array}$ \\
\hline 3 & Definido & Controle & $\begin{array}{c}\text { Padronização Organizacional } \\
\text { e Processos Institucionais }\end{array}$ & Metodologia Singular & Padronizado & $\begin{array}{c}\text { Processo } \\
\text { Definido }\end{array}$ \\
\hline 4 & Gerenciado & $\begin{array}{c}\text { Melhoria } \\
\text { Contínua }\end{array}$ & Processo Gerenciado & Benchmarking & Gerenciado & $\begin{array}{c}\text { Processo } \\
\text { Gerenciado }\end{array}$ \\
\hline 5 & Otimizado & - & Processo Otimizado & Melhoria Contínua & Otimizado & $\begin{array}{c}\text { Processo } \\
\text { Otimizado }\end{array}$ \\
\hline
\end{tabular}

Fonte: Costa \& Moura (2009, p. 42)

de entregas, metas de prazos e orçamentos e a excelência na GP (Kerzner, 2011).

Os projetos podem ser considerados importantes instrumentos de mudança e de desenvolvimento das organizações (Bouer \& Carvalho, 2005); com isso, a GP cria vantagem competitiva (Shenhar, 2004) e torna-se uma capacidade essencial para o sucesso (Shenhar \& Dvir, 2010). As empresas buscam as melhores práticas em GP que contribuam para o planejamento, execução e o controle dos recursos da empresa (Kerzner, 2011). A melhoria contínua deve ser buscada incansavelmente, portanto, para compreender a eficácia da gestão do projeto de uma organização é determinante a sua maturidade em GP (PMM). A maturidade na GP está associada a uma metodologia padrão de processos de acompanhamento, contribuindo para o sucesso do projeto (Rabechini, 2005; Kerzner, 2006, 2009).

A literatura apresenta diversos modelos de maturidade (Carvalho \& Rabechini, 2011), capazes de quantificar numericamente a maturidade. Dinsmore (1999) explica que a maturidade reflete o quanto uma organização avançou em relação à incorporação das práticas de GP, contribuindo para eficácia em concluir os mesmos. Assim, a maturidade em GP está ligada a quão hábil uma organização está no exercício de gerenciar seus projetos (Prado, 2004). Cada empresa, a partir das experiências e do aprendizado, faz uso de técnicas e ferramentas de GP, capazes de refletir na maturidade de seus processos (Dinsmore \& Cabanis-Brewin, 2006).

A Tabela 1 apresenta uma análise comparativa entre modelos de maturidade baseados no modelo percursor CMM (Costa \& Moura, 2009).

Lopes (2009) aponta 20 características da GP que influenciam a maturidade e o sucesso do projeto: (1) desempenho de prazo e cronograma; (2) desempenho de escopo; (3) desempenho de custo; (4) planejamento de tempo; (5) planejamento de escopo; (6) planejamento de custo; (7) definição de requisitos; (8) atendimento a requisitos técnicos; (9) definição de objetivos e estratégias; (10) gerenciamento de mudança; (11) gerenciamento de riscos; (12) gerenciamento da comunicação; (13) gerenciamento de encerramento; (14) liderança e relacionamento; (15) delegação de responsabilidades; (16) ética profissional de gerentes de projetos; (17) tomada de decisões; (18) envolvimento dos stakeholders; (19) controle e monitoramento; e (20) sustentabilidade do projeto.

Alguns autores afirmam que o sucesso do negócio depende de projetos (Shenhar \& Dvir, 2010), e as empresas que adotam a prática madura de GP estão mais capacitadas ao sucesso (Kerzner, 2006). Os modelos de maturidade em GP permitem a realização de um processo de aprendizagem das práticas de GP adotadas pela organização e, consequentemente, a melhoria das competências em GP (Carvalho \& Rabechini, 2011; Kerzner, 2006). A gestão por projetos é avaliada por muitas empresas como uma ferramenta importante para lidar com o crescimento e garantir a sobrevivência das empresas em função da sua utilidade para aperfeiçoar a alocação dos recursos (Sbragia, Rodrigues, Piscopo \& Moreira, 2009). Nesse sentido, ela pode também ser útil para os processos de internacionalização.

\subsection{Internacionalização de empresas}

Partindo da análise da GP para a internacionalização de empresas, um pressuposto básico que conduzirá esta análise é a existência de imperfeições de mercado (Moreira, 2009), o que corrobora com o 
estudo de projetos, pois as imperfeições do mercado o tornam um ambiente complexo e dinâmico para atuação das empresas (Porter, 1993; Hitt et al., 2011). As empresas mais competitivas têm melhor desempenho e posicionamento no mercado (Porter, 1996; Kotler, 1998).

A internacionalização refere-se ao grau em que uma empresa obtém suas receitas ou operações de vendas realizadas fora do seu país de origem (Elango \& Pattnaik, 2007); também pode ser caracterizada como um processo crescente e continuado de envolvimento de uma empresa nas operações com outros países (Goulart, et. al. 1994); e, ainda, pelo envolvimento e comprometimento das operações internacionais da empresa ao longo do tempo (Kraus, 2006). O processo de internacionalização tem sido uma das estratégias utilizadas pelas empresas como forma de crescimento, seja para acessar novos mercados, manter a produtividade industrial combatendo as sazonalidades internas, aquisição de novos produtos, insumos e tecnologias, ou para aproveitar novas oportunidades de negócios (Dunning, 2001; Floriani, 2010).

Existem diversas teorias e modelos do processo de internacionalização de empresas, em diversas óticas e argumentos (Vianna, Piscopo \& Ryngelblum, 2013). Embora essas diferentes abordagens, há certo consenso, segundo os autores, de que o processo de entrada em mercados internacionais promove mudanças significativas nas empresas. A decisão de internacionalizar é considerada estratégica, pois envolve responder questões do tipo: o que, por que, onde, como, quando e quanto (Vianna et al., 2013; Madeira \& Silveira, 2013).

De acordo com a abordagem de estágio, as empresas começam a venda de produtos em seus mercados domésticos e, em seguida, gradualmente buscam novos países. Podem ser identificados dentro da abordagem estágio dois modelos principais: a Teoria Ciclo de Vida do Produto de Raymond Vernon (1966) e o Modelo de Internacionalização de Uppsala (Johanson \& Wiedersheim-Paul, 1975; Johanson \& Vahlne, 1977, 1990).

O processo de internacionalização pode ser dividido em duas correntes teóricas: a teoria de abordagem econômica e a teoria de abordagem comportamental (Almor, Hashai \& Hirsch, 2006; Floriani, 2010; Vianna et al., 2013; Madeira \& Silveira, 2013). As teorias de abordagem econômica têm sua base na corrente principal de economia, e centram- se na relação entre a empresa e seu ambiente (Andersson, 2000; Madeira \& Silveira, 2013); seu principal aspecto é que as empresas são quase racionais em sua escolha de investimentos e o decisor tem acesso à informação (Andersson, 2000). As teorias de abordagem comportamental tratam da aprendizagem individual dos gestores, como aspectos importantes na compreensão do comportamento internacional (Andersson, 2000); o foco é no impacto da experiência internacional sobre o ritmo e a direção do processo de internacionalização.

Diversas questões de natureza externa ou interna podem influenciar o desempenho do processo de internacionalização. No aspecto interno estão as questões relacionadas à própria organização, e no externo o mercado e o ambiente empresarial (Douglas \& Craig, 1995). Outra questão associada à decisão de internacionalizar é a escolha do modo de entrada apropriado. O modo de entrada é um determinante crítico do envolvimento e provável sucesso internacional da organização, que varia de acordo com o comprometimento e a complexidade da operação, podendo ser realizado por meio da exportação, licenciamento da marca, aliança estratégica, aquisições e a implantação de uma nova subsidiária (Root, 1987; Hitt et al., 2011).

Assim, o processo de internacionalização sofre influências de aspectos ligados ao ambiente interno (estrutura, recursos, competências, entre outros) e ao ambiente externo (regulação, cultura, barreiras, etc.), da mesma forma que ocorre com os projetos. Com isso, a internacionalização de empresas engloba (Moreira, 2009): confrontos estáticos - tipos de operações, seus compromissos e os conhecimentos que a empresa detém sobre determinado mercadoalvo; e dinâmicos - a evolução das atividades internacionais da empresa, os riscos associados a determinados mercados e a falta de experiências nesses mercados. As influências do ambiente em que as empresas estão inseridas e competem também contribuem para promoção ou não da vantagem competitiva (Porter, 1993), além dos recursos estratégicos desenvolvidos pela empresa. Além disso, a internacionalização das empresas pode ocorrer em decorrência de vantagens competitivas no mercado doméstico (Goulart et al. 1994).

A implicação competitiva do uso das práticas maduras da GP, como premissa para expandir internacionalmente, é analisada neste estudo a partir 
da perspectiva da RBV, tema que será abordado a seguir.

\subsection{Visão Baseada em Recursos - RBV}

A RBV fornece insumos para analisar o comportamento estratégico internacional da empresa, concentrando-se sobre o conjunto de recursos e competências únicas que pertencem ou que tenham sido adquiridos externamente pelas empresas (Penrose, 2006; Barney, 1991). Combinar de forma intencional, racionalmente, os recursos disponíveis na empresa é uma ação estratégica, cumprindo metas para o alcance de objetivos organizacionais num determinado mercado, obtendo com isso a vantagem competitiva (Hatch, 1997).

Um dos maiores objetivos das empresas é a sobrevivência em condições sustentáveis, com perspectivas de crescimento, sendo a criação de recursos e capacidades elementos indispensáveis para sua consecução da vantagem competitiva sustentável (Wernerfelt 1984; Barney, 1991; Peteraf 1993). Além disso, nenhuma empresa pode sobreviver no mercado sem a existência dos recursos (Grant, 1991), sendo estes como a unidade básica que fornece às empresas a entrada no processo dos negócios (Hollensen, 2011). A RBV é uma estrutura teórica no campo da gestão estratégica, usada para entender a vantagem competitiva da empresa e sua sustentabilidade ao longo do tempo (Barney, 1991; Barney et al. 2007).

De acordo com a RBV, os recursos estratégicos de posse da empresa e os recursos disponíveis no setor, são a fonte de vantagens competitivas sustentáveis. Assim, os recursos da empresa são mais importantes que os recursos no setor. Com isso, as relações entre os recursos e a posição da empresa no mercado estão muito próximas. O tamanho da empresa está ligado ao tamanho da influência das forças de mercado para a destinação e as diferentes formas de uso dos seus recursos, ou seja, quanto maior o tamanho da empresa, menor será a influência (Penrose, 2006).

Inicialmente, a vantagem competitiva sustentável da empresa baseava-se na análise de quatro características que potencializavam os recursos de uma empresa: valor, raridade, difícil imitação, e difícil substituição (Barney, 1991). Posteriormente, essas características surgem de uma forma levemente alterada, sendo os três primeiros mantidos, e a última alterada (Barney \& Clark, 2007). Com isso, os recursos e capacidades assumem quatro características distintas: valioso, raro, imitável e organizável - VRIO (Barney, 2001; Barney \& Clark, 2007).

As características apresentadas pelo modelo VRIO possibilitam realizar análise baseada em recursos com perguntas sobre as atividades dos negócios da empresa (Barney \& Clark, 2007): (1) Valor - Os recursos e capacidades permitem que a empresa responda às ameaças ou oportunidades? (2) Raridade - O recurso é atualmente controlado por um pequeno número de concorrentes? (3) Imitabilidade (difícil imitação) - As empresas sem os recursos enfrentam uma desvantagem de custo para obtê-lo ou desenvolvê-lo? (4) Organização - A empresa possui políticas e procedimentos organizados para apoiar a exploração de seus recursos valiosos, raros e custosos para imitar? Essas questões possibilitam compreender o potencial de retorno associado ao uso de qualquer recurso ou capacidade de uma empresa (Barney \& Clark, 2007), conforme representado na Tabela 2 ).

A abordagem da RBV pode contribuir para compreensão de como os recursos são empregados na internacionalização e como podem conferir vantagem competitiva no mercado internacional.

Tab. 2

VRIO Model

\begin{tabular}{cccccc}
\hline \multicolumn{5}{c}{ É um recurso ou capacidade... } \\
\hline Valioso? & Raro? & Difícil & Valioso? & $\begin{array}{c}\text { Raro? } \\
\text { Desvantagem } \\
\text { Competitiva }\end{array}$ & Difícil \\
Não & - & - & Não & Paridade Competitiva do Normal & Normal \\
$\operatorname{Sim}$ & Não & - & Vantagem Competitiva & Acima do Normal \\
$\operatorname{Sim}$ & Sim & Não & Temporária & Vantagem Competitiva & Acima do Normal \\
$\operatorname{Sim}$ & $\operatorname{Sim}$ & $\operatorname{Sim}$ & $\operatorname{Sim}$ & Sustentável &
\end{tabular}

Fonte: Costa \& Moura (2009, p. 42) 


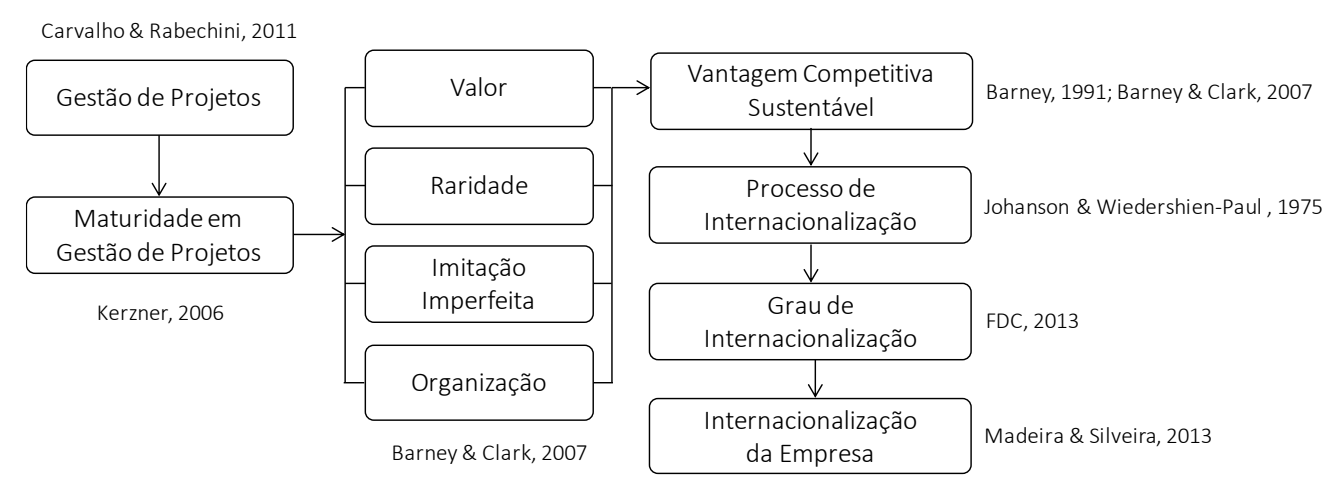

Fig. 1

Modelo Teórico da pesquisa

Fonte: Elaborado pelos autores

\subsection{Relação entre Gestão de Projetos, Internacionalização e RBV}

Estudos evidenciam que algumas características organizacionais influenciam a expansão internacional das empresas em determinadas dimensões, interferindo no grau de internacionalização das empresas (Hitt, Hoskissom \& Kim, 1997; Barretto \& Rocha, 2003). Essas características organizacionais são os recursos da empresa, dos quais conferem uma vantagem para empresa, contribuindo para o processo de entrada em mercados específicos (Madhok, 1997; Hollensen, 2011).

Para o desenvolvimento deste estudo, a GP foi analisada como um recurso estratégico interno da empresa, por meio do VRIO, que consequentemente, pode conferir uma vantagem competitiva sustentável para a internacionalização da empresa, com base na RBV. A internacionalização da empresa, por sua vez, foi analisada com base no processo de internacionalização (Por quê? Como? Quando? Onde? O que?).

A Figura 1 apresenta o modelo teórico da pesquisa. A perspectiva do modelo é investigar essa relação do ponto de vista da gestão de projeto ser um recurso estratégico que confere vantagem competitiva sustentável para as empresas que se utilizam dela, sob o olhar das variáveis VRIO da RBV. Com isso, sugere-se que o nível de maturidade da empresa na execução de seus projetos contribui para o desempenho da internacionalização da empresa.

A seguir, será apresentado o método de pesquisa.

\section{MÉTODO DE PESQUISA}

A estratégia metodológica adotada foi o estudo de caso múltiplo (Eisenhardt, 1989). Trata-se de uma pesquisa descritivo-exploratória, de abordagem qualitativa, pois foram relatadas as evidências do comportamento das empresas selecionadas

Tab. 3

Coleta de dados

\begin{tabular}{|c|c|c|}
\hline Investigação & Modelo & Procedimento \\
\hline Maturidade em GP & KPMMM & $\begin{array}{l}\text { Optou-se por utilizar um questionário estruturado já testado para avaliar o nível } 2 \text { (Processos } \\
\text { Comuns) da maturidade em GP (Bouer \& Carvalho, 2005), seguindo o modelo de maturidade } \\
\text { KPMMM (Kerzner, 2006). O questionário foi respondido por } 15 \text { pessoas, sendo } 4 \text { na empresa Alpha, } 6 \\
\text { na empresa Beta, e } 5 \text { na empresa Zeta. }\end{array}$ \\
\hline $\begin{array}{r}\text { Processo de } \\
\text { Internacionalização }\end{array}$ & Uppsala & $\begin{array}{l}\text { Utilizou-se um questionário semiestruturado baseado no Modelo Incremental de Uppsala (Johanson } \\
\text { \& Vahlne, 1977, 1990), alinhando ainda as motivações estratégicas para internacionalização da } \\
\text { empresa (Lientz \& Rea, 2003; Hitt et al., 2011). O questionário foi respondido por } 3 \text { pessoas, sendo } 1 \\
\text { por empresa. }\end{array}$ \\
\hline $\begin{array}{r}\text { Grau de } \\
\text { Internacionalização da } \\
\text { Empresa }\end{array}$ & UNCTAD & $\begin{array}{l}\text { Utilizou-se o cálculo do índice de transnacionalidade no exterior, desenvolvido pela UNCTAD, e } \\
\text { utilizado no Brasil pela FDC. O cálculo leva em consideração: os ativos, as receitas e o número de } \\
\text { funcionários das empresas (FDC, 2013, p.11). }\end{array}$ \\
\hline Implicação Competitiva & RBV-VRIO & $\begin{array}{l}\text { Utilizou-se um questionário estruturado relacionando às características de maturidade em GP (Lopes, } \\
\text { 2009) avaliando a implicação competitiva das características à luz das dimensões da RBV-VRIO } \\
\text { (valioso, raro, imitável e suportado pela organização), para internacionalização (Barney \& Clark, } \\
\text { 2007). O questionário foi respondido por } 3 \text { pessoas, sendo } 1 \text { por empresa. }\end{array}$ \\
\hline
\end{tabular}

Fonte: Elaborado pelos Autores

Internext | São Paulo, v.12, n. 3, p. 01-15, set./dez. 2017 
Tab. 4

Matriz de Correlação: Gestão de Projetos x RBV (VRIO) x Internacionalização da Empresa

\begin{tabular}{|c|c|c|c|}
\hline $\begin{array}{c}\text { Critério GP } \\
\text { (Recursos e Capacidades) }\end{array}$ & $\begin{array}{c}\text { Características RBV } \\
\text { - VRIO - }\end{array}$ & Implicação Competitiva & $\begin{array}{l}\text { Contribuição para } \\
\text { Internacionalização }\end{array}$ \\
\hline $\begin{array}{c}\text { Desempenho de Prazo e Cronograma } \\
\text { Desempenho de Escopo } \\
\text { Desempenho de Custo } \\
\text { Planejamento de Tempo }\end{array}$ & É valioso? & Desvantagem Competitiva & \multirow[b]{2}{*}{$\begin{array}{l}\text { Contribuí para o Processo de } \\
\text { Internacionalização? }\end{array}$} \\
\hline $\begin{array}{c}\text { Planejamento de Escopo } \\
\text { Planejamento de Custo } \\
\text { Definição de Requisitos } \\
\text { Atendimento a Requisitos Técnicos }\end{array}$ & É raro? & Paridade Competitiva & \\
\hline $\begin{array}{c}\text { Definição de Objetivos e Estratégias } \\
\text { Gerenciamento de Mudança } \\
\text { Gerenciamento de Riscos }\end{array}$ & \multirow[b]{2}{*}{ É difícil de Imitar? } & & \multirow{3}{*}{$\begin{array}{l}\text { Não contribuí para o Processo } \\
\text { de Internacionalização? }\end{array}$} \\
\hline $\begin{array}{l}\text { Gerenciamento da Comunicação } \\
\text { Gerenciamento de Encerramento } \\
\text { Liderança e Relacionamento } \\
\text { Delegação de Responsabilidades } \\
\text { Ética Profissional de Gerentes de Projetos }\end{array}$ & & Vantagem Temporária & \\
\hline $\begin{array}{l}\text { Tomada de Decisões } \\
\text { Envolvimento dos Stakeholders } \\
\text { Controle e Monitoramento } \\
\text { Sustentabilidade do Projeto }\end{array}$ & $\begin{array}{l}\text { É explorado pela } \\
\text { Organização? }\end{array}$ & Vantagem Sustentável & \\
\hline
\end{tabular}

Fonte: Elaborado pelos Autores com base em Barney \& Clark, 2007.

explorando a contribuição da maturidade na gestão dos projetos para internacionalização das empresas sob a ótica do RBV, sem interferência do pesquisador.

Três empresas foram foco do estudo: a primeira do setor de atacado, no segmento do agronegócio; a segunda do setor de bens de capital, no segmento de máquinas e equipamentos; e a terceira no setor de bens de consumo, no segmento de alimentos. A definição das unidades de análise foi feita de forma intencional e por conveniência, tendo como base os seguintes critérios: a) ter origem brasileira, mesmo que controlada por grupos internacionais; b) ser de grande porte, e considerada líder em seu segmento de atuação; c) empresas de segmentos diferentes; d) possuir regularidade nos negócios internacionais por pelo menos 3 anos; e) fazer uso da GP. O foco da análise foram as operações internacionais das empresas, considerando-se dados do grupo. A identificação das empresas foi mantida em sigilo, sendo aqui denominadas como Alpha, Beta e Zeta.

A Tabela 3 apresenta os procedimentos para coleta de dados, baseada em instrumentos provenientes da literatura:

A análise dos dados envolveu a análise intracaso e intercaso (Eisenhardt, 1989). Iniciou-se pela narrativa geral dos dados coletados, triangulação com as diversas fontes de evidências (relatórios, balanços, publicações, websites e outros documentos), passando para uma síntese geral dos dados. Adotouse o ano de 2013 como referência para a análise do contexto em virtude de as empresas disponibilizarem os dados consolidados, receitas e despesas, e seus relatórios de gestão desse ano.

No contexto da GP, optou-se pela avaliação do Modelo KPMMM (Kerzner, 2006), nível 2 de maturidade - Processos Comuns, mostrou-se adequado, pois a maioria das empresas ainda não alcançaram os níveis 3, 4 e 5, que tradicionalmente, são alcançados por empresas em que a natureza das suas atividades exige uma atenção e dedicação maior, tais como: aeroespacial, petrolífero, petroquímico, construção civil, armamento militar e telecomunicações (Bouer \& Carvalho, 2005). Esta avaliação da maturidade considera aspectos da GP relacionados ao uso de técnicas de gestão de escopo, custo e prazo, como também se existe a figura do gerente de projeto na empresa.

No contexto da internacionalização considerou-se o comportamento ao longo do tempo, realizando entrevista em questionário semiestruturado baseado no modelo incremental de Uppsala (Johanson \& Vahlne, 1977, 1990), alinhando ainda as motivações estratégicas para internacionalização da empresa (Andersen, 1993; Lientz \& Rea, 2003; Hitt et al., 2011). Foram utilizados relatórios de gestão e balanços, de forma complementar. Para avaliação da GP como recursos estratégicos para empresa foi utilizada a matriz de correlação, baseado na análise das características de sucesso em projetos (Lopes, 2009) sob as dimensões da RBV para alcance da 
Tab. 5

Principais dados das empresas selecionadas.

\begin{tabular}{cccc}
\hline Características & Empresa Alpha & Empresa Beta & Empresa Zeta \\
\hline Tipologia Jurídica & $($ Ltda.) & (S.A.) & (S.A.) \\
Setor de Mercado & Atacado & Bens de Capital & Bens de Consumo \\
Segmento Empresarial & Agronegócios & Máquinas e Equipamentos & Alimentos \\
Porte da Empresa & Grande Empresa & Grande Empresa & Grande Empresa \\
Funcionários & 3.949 & 29.099 & 1.958 \\
Valor Exportado em 2013 & Acima US\$ 100 milhões & Acima US\$ 100 milhões & Até US\$ 50 milhões \\
Atuação Internacional & Desde 2000 & Desde 1970 & Desde 2000 \\
Quantidade de Países & 4 países & 28 países & 3 países \\
Escritório de Projetos & Não & Sim & Não \\
Estado Sede & Mato Grosso & Santa Catarina & Santa Catarina \\
\hline
\end{tabular}

Fonte: Elaborado pelos autores.

vantagem competitiva sustentável (Barney \& Clark, 2007). Esta matriz considerou quatro questões chaves no modelo RBV-VRIO (Barney \& Clark, 2007): (1) A questão do Valor - Os recursos e capacidades permitem que a empresa responda às ameaças ou oportunidades ambientais?; (2) A questão da Raridade - O recurso é atualmente controlado por apenas um pequeno número de empresas concorrentes?; (3) A questão da difícil imitação - Será que as empresas sem os recursos enfrentam uma desvantagem de custo para obtê-lo ou desenvolvêlo?; e (4) A questão da Organização - A empresa possui políticas e procedimentos organizados para apoiar a exploração de seus recursos valiosos, raros e custosos para imitar?

Os instrumentos de coleta de dados foram testados durante a etapa de validação do instrumento. Na sequência, foi aplicado em formato eletrônico tendo-se obtido respostas de 5 pessoas na empresa Alpha, 7 na Beta e 6 na Zeta (todos relacionados à GP). Também foi realizada a entrevista com os executivos da área internacional de cada caso (um por empresa). O modelo proposto permitiu analisar essas características da GP como recursos e capacidades conforme apresentado na Tabela 4.

O estudo considerou ainda a transnacionalidade das empresas com intuito de complementar a análise sob a ótica da internacionalização. Essa análise considera o resultado obtido do cálculo do índice de transnacionalidade, desenvolvido pela UNCTAD e utilizado pela FDC no Brasil. O cálculo leva em consideração: os ativos, as receitas e o número de funcionários das empresas (Figura 2).

$\underline{\text { Ativos no Exterior }}+\frac{\text { Receitas no Exterior }}{\text { Ativos Totais }}+\frac{\text { Funcionários no Exterior }}{\text { Funcionarios Totais }}$ $\frac{\frac{\text { Ativos Totais }}{\text { Receitas Totais }}+\frac{\text { Funcionários Totais }}{3}}{3}$

Fig. 2

Fórmula de cálculo do índice de transnacionalidade no exterior. Fonte: FDC (2013, p. 11).
O tempo para realização de todos os protocolos foi de cerca de 2 horas por empresa, considerando a etapa on-line (múltiplos respondentes) e a etapa presencial (entrevista com único respondente), e para as anotações dos comentários e observações mais vinte minutos. Foram utilizadas ainda diversas fontes de evidências para uma triangulação dos dados, sendo: (i) $\mathrm{Na}$ Empresa Alha, utilizados relatório de gestão, relatório da fundação da empresa, relatório de sustentabilidade, relatório do projeto de expansão, relatório financeiro com volume de negócios, revistas, releases e website; (ii) $\mathrm{Na}$ Empresa Beta, utilizados relatório integrado de gestão, livro dos 50 anos da empresa, balanço financeiro, relatório de dados cadastrais das empresas do grupo, revistas, releases e websites; e (iii) Na Empresa Zeta, utilizados relatórios de gestão, relatório $\mathrm{GRI}$, relatório financeiro, revistas, releases e websites. A intenção foi de acessar o maior número de gerentes de projetos em cada empresa, e pelo menos um respondente da alta administração para aplicação dos questionários e realização das entrevistas.

\section{ANÁLISE DOS RESULTADOS}

As empresas foco deste estudo (Tabela 5) atuam em setores empresariais altamente competitivos, onde as negociações são decididas com pequenas variações de preço. Mesmo no ambiente macroeconômico internacional em crise nos anos de 2008 e 2009, as três empresas continuaram com foco no mercado internacional, com decisões estratégicas para internacionalização deliberadas e planejadas. Os três casos apresentam elementos que evidenciam as práticas de GP, no entanto apenas a Empresa Beta possui estrutura organizacional mais projetizada, com escritório de projetos estruturado.

A partir dos resultados, foi realizada uma análise comparativa das empresas estudadas, com base em

Internext | São Paulo, v.12, n. 3, p. 01-15, set./dez. 2017 
três variáveis que pudessem equilibradamente compará-las, considerando que são de segmentos empresariais e portes diferentes e com uma presença internacional distinta. As três variáveis foram: (1) análise da maturidade, utilizando a pontuação alcançada por cada empresa na fase de maturidade do modelo KPMMM; (2) análise da internacionalização, utilizando o índice de transnacionalidade obtido por meio da fórmula que envolve o cálculo dos ativos, receitas e funcionários das empresas; e (3) análise da implicação competitiva com contribuição para internacionalização, utilizando o coeficiente do número de vezes que as três empresas apresentaram as mesmas características maduras na GP com vantagem competitiva sustentável, contribuindo para internacionalização das empresas.

Do ponto de vista da análise da maturidade em GP, verifica-se que as três empresas possuem pontuações distintas, no entanto a fase embrionária é a única fase em que as empresas apresentam praticamente as mesmas pontuações. Uma elevada pontuação na fase embrionária pressupõe que as empresas compreendem e perfilham os benefícios da GP, sobretudo nas estruturas operacionais e de supervisão (Bouer \& Carvalho, 2005). A Figura 3 apresenta a pontuação média alcançada das áreas técnicas investigadas nas empresas, para avaliação da maturidade na GP.

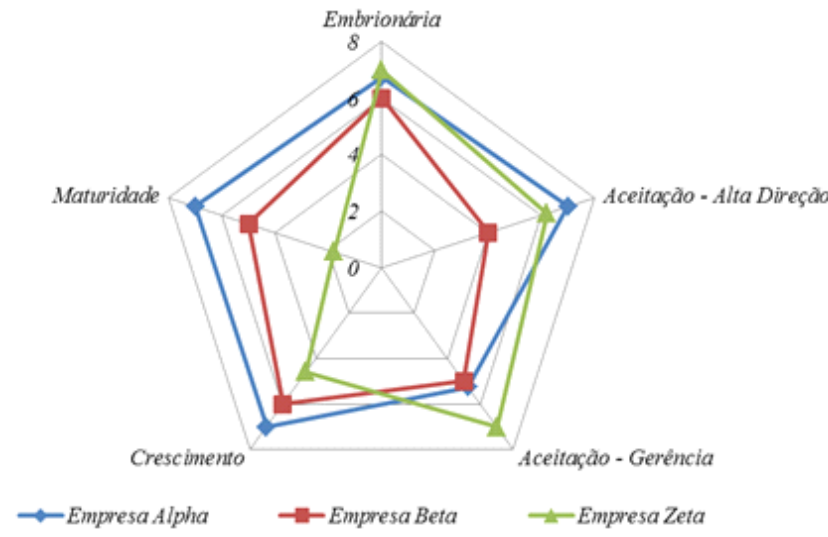

Fig. 3

Análise Comparativa da Avaliação da Maturidade na GP das Empresas

Fonte: Elaborado pelos autores.

Analisando os resultados, a Empresa Alpha apresenta um bom desempenho na avaliação do seu nível de maturidade em GP, pois obteve pontuação 7 (pontuação máxima 12) nas fases Embrionária, Aceitação - Alta Direção, Crescimento e Maturidade, tendo apenas nota 5 na fase Aceitação - Gerência.
Parece ser um reflexo natural do seu modelo de negócio, considerando que a empresa dependente de processos e cronogramas controlados em toda a atividade produtiva. Esse desempenho na avaliação sugere que a empresa esteja acima do nível 2 de maturidade, podendo estar entre o Nível 3 Metodologia Singular, e o Nível 4 - Benchmarking, marcada pela elevada pontuação nas fases de Crescimento e Maturidade, ambas com nota 7. A fase de Crescimento é crucial para empresa, pois marca o começo do desenvolvimento de um processo de GP, sendo o foco principal nesse caso, padronizar os métodos de planejamento, execução e controle dos projetos (Kerzner, 2009).

A Empresa Beta possui um desempenho adequado e equilibrado em suas pontuações de maturidade na GP. A empresa obteve pontuação 6 na fase Embrionária, 4 na fase Aceitação - Alta Direção, 5 na fase Aceitação - Gerência, 6 na fase Crescimento e 5 na fase Maturidade. Mesmo com grande variação nos resultados obtidos, a Empresa Beta conseguiu manter-se próxima da média 6 na pontuação geral. Sua estrutura está organizada de forma a atender a gestão por projetos e por operações. Na gestão por operações estão definidos claramente processos, rotinas e produtos que são produzidos para atender os lotes e pedidos de entrega, basicamente pela produção em série das máquinas, equipamentos, peças e outros produtos fabricados especialmente para atender grandes empresas, com custo inferior aos encontrados no mercado doméstico. Na gestão por projetos a estrutura do negócio canaliza os esforços para a pesquisa e desenvolvimento de novas soluções e aplicações tecnológicas, o estímulo à inovação. Nesse modelo, o atendimento é voltado para os projetos especiais, com contratos específicos como licitações, concorrências públicas, cadeias produtivas com soluções e aplicações customizadas e desenhadas de acordo com a necessidade do cliente, nesses casos a agregação do valor aos serviços é maior possibilitando maior rentabilidade. Essa configuração organizacional contribui para o desempenho equilibrado na avaliação da maturidade da Empresa Beta, sinalizando que a empresa esteja no Nível 3 de Maturidade em GP, Metodologia Singular.

A Empresa Zeta seguiu com elevada pontuação nas fases Embrionária (7), Aceitação - Alta Direção (6) e Aceitação - Gerência (7), ao passo que na fase de Crescimento (5) e Maturidade (2) obteve baixa pontuação. Pode-se sugerir que a Empresa Zeta 
percebe e reconhece os benefícios da GP, principalmente nos níveis operacionais e de supervisão da empresa. Além disso, a elevada pontuação nas fases 2 e 3 significa que os conceitos e as práticas da GP são amplamente aceitos, tornandose aparentes o apoio e o empenho das lideranças da Empresa Zeta no patrocínio e condução dos projetos. Como houve uma tendência de baixa pontuação a partir da fase de Crescimento, e se confirmou com a pontuação da fase de Maturidade, significa que a empresa ainda busca a padronização de uma metodologia para o planejamento, execução e controle dos seus projetos. Na sequência, a Maturidade não foi alcançada. Podem-se relacionar diversos fatores para $\mathrm{o}$ não atingimento da Maturidade, como a resistência da empresa em efetuar rígidos controles de prazos e custos, com relatórios periódicos e sistematizados, o desenvolvimento de uma grade de competência e habilidades em GP e a profissionalização da função de GP como carreira (Bouer \& Carvalho, 2005). Esses resultados indicam que a Empresa Zeta ainda esteja no Nível 2 - Processos Comuns, de Maturidade em GP.

Para medir a presença internacional adotou-se o índice de transnacionalidade. Os resultados obtidos pelos cálculos foram: (a) Empresa Alpha com índice de 0,153; (b) Empresa Beta com índice de 0,382; e (c) Empresa Zeta com índice de 0,130. Comparativamente a diferença nos índices não significa que uma empresa é mais atrasada que a outra na estratégia de internacionalização, podendo significar, por exemplo, que uma empresa tem maior participação no mercado interno em relação à outra. O índice apresenta a proporção da atuação no exterior com relação ao volume total das operações (FDC, 2013).

A análise da implicação competitiva RBV-VRIO nas empresas tomou como base o cálculo do coeficiente alcançado a partir do número de vezes em que cada empresa apresentou características maduras na GP com alta contribuição para internacionalização com implicação de vantagem competitiva sustentável. Os resultados foram: (i) Empresa Alpha apresentou 11 características, representando $55 \%$ das 20 características; (ii) Empresa Beta apresentou 17 características, representando $85 \%$ das 20 características; e (iii) Empresa Zeta apresentou 10 características, representando $50 \%$ das 20 características. Esses resultados demonstram que a Empresa Beta possui maior vantagem competitiva sustentável que as demais no uso da GP como um recurso estratégico para sua expansão internacional.

Tab. 6

Matriz de Avaliação:

Maturidade $x$ Transnacionalidade $x$ RBV-VRIO

\begin{tabular}{cccc}
\hline EMPRESA & MATURIDADE & TRANSNACIONALIDADE & RBV-VRIO \\
\hline Alpha & 7.00 & 0.153 & $55 \%$ \\
Beta & 5.00 & 0.382 & $85 \%$ \\
Zeta & 2.00 & 0.130 & $50 \%$ \\
\hline
\end{tabular}

Fonte: Elaborado pelos autores.

A Tabela 6 apresenta a matriz de avaliação das empresas estudadas na pesquisa comparando as três variáveis: maturidade na $G P$, transnacionalidade e RBV-VRIO.

Verificou-se que apesar da Empresa Alpha possui maior maturidade na GP em relação às demais empresas, ela não conseguiu converter a prática madura da GP em vantagem competitiva sustentável, obtendo apenas 55\% das características RBV-VRIO, e ainda com o índice de transnacionalidade entre a Empresa Beta e Zeta. O mesmo ocorre com a Empresa Zeta, no entanto o caso dela é ainda mais evidente, pois não obteve uma boa pontuação na fase de maturidade e apresenta apenas a metade das características maduras da GP com implicação de vantagem competitiva sustentável, com menor índice de transnacionalidade. A Empresa Beta apresenta o melhor resultado com uma boa pontuação na maturidade, um alto índice de transnacionalidade e com $85 \%$ das características da maturidade na GP aplicados para internacionalização com implicação de vantagem competitiva sustentável.

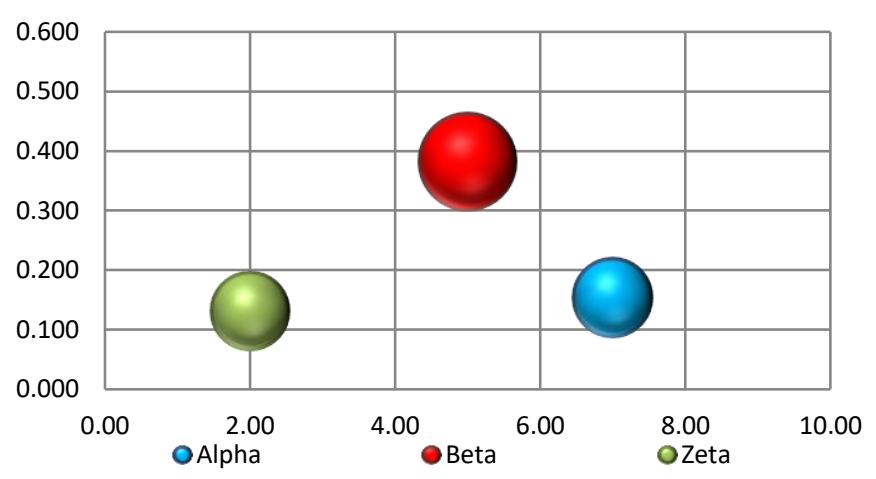

Fig. 4

Matriz de Avaliação:

Maturidade x Transnacionalidade x RBV (VRIO)

Fonte: Elaborado pelos autores

A Figura 4 apresenta uma perspectiva espacial do posicionamento das empresas nas três variáveis. $\mathrm{O}$ Eixo $X$ representa o nível de maturidade, o Eixo Y o 


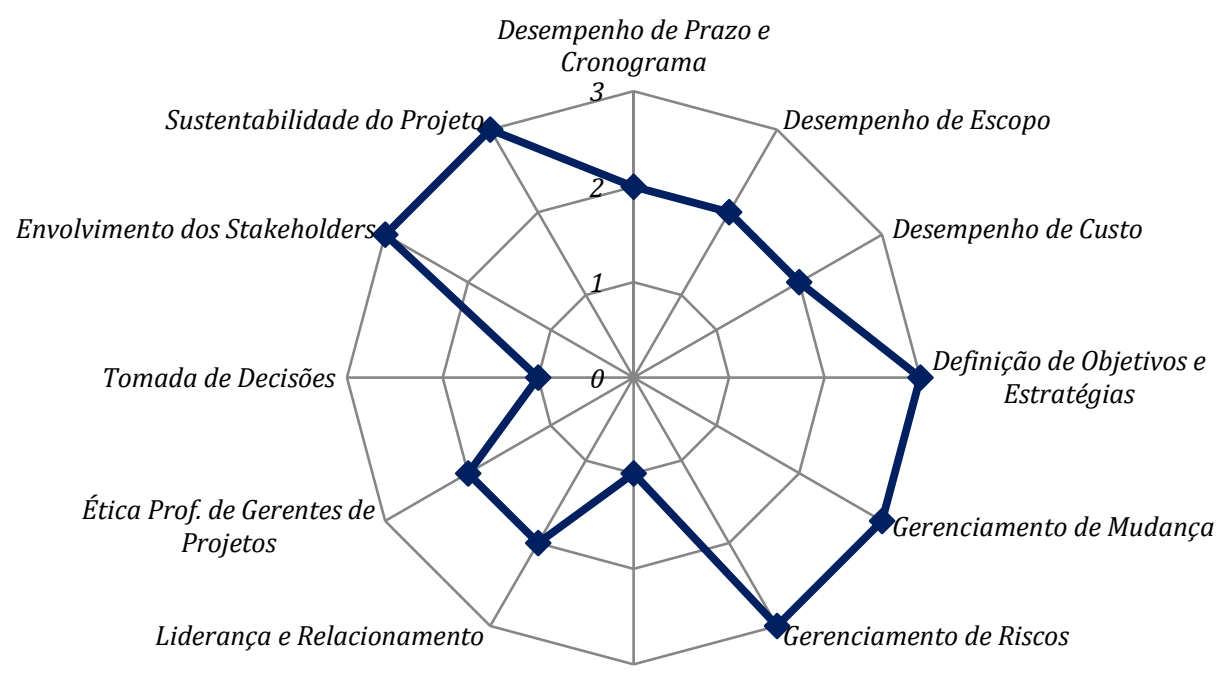

Gerenciamento da Comunicação

$\longrightarrow$ Ocorrências

Fig. 5

Características da GP com maior contribuição para internacionalização com implicação vantagem competitiva sustentável.

Fonte: Elaborado pelos autores.

índice de transnacionalidade, e o tamanho das esferas o percentual das características RBV-VRIO.

Com base na análise dessa matriz, sugere-se que em relação aos três casos estudados, a simples utilização das características da gestão madura de projetos não implica em uma maior internacionalização, pois o nível 2 (processos comuns) analisado considera apenas as variáveis de escopo, custo e prazo, o qual são condições fundamentais para o início na maturidade em GP. No entanto, algumas características da GP foram identificadas com maior contribuição para o processo de internacionalização com implicação de vantagem competitiva sustentável. Outros aspectos precisam ser avaliados, como o cenário econômico, a disponibilidade de recursos financeiros nas empresas, oportunidades mercadológicas, entre outros aspectos. No entanto, considerando os resultados obtidos na pesquisa e analisando a Figura 4 (características da GP com maior contribuição para internacionalização com implicação de vantagem competitiva sustentável) pode-se concluir que a Empresa Beta utiliza com maior propriedade as características da gestão madura de projetos para o alcance da sua vantagem competitiva sustentável repercutindo no melhor posicionamento na transnacionalidade.

Dessas 12 características, apenas 5 foram comuns aos três casos estudados. A partir disso, foram mapeadas as 5 características da gestão madura de projetos com maior contribuição para internacionalização que confere vantagem competitiva sustentável para os três casos estudados.

\section{CONCLUSÕES}

Os resultados do estudo sugerem que cinco características da gestão madura de projetos (recursos) contribuem para a internacionalização das empresas estudadas, proporcionando uma vantagem competitiva sustentável no mercado, corroborando a revisão da literatura no sentido de que uma empresa desenvolve vantagem competitiva a partir de recursos estratégicos. As principais mudanças organizacionais e as iniciativas para gerar vantagens competitivas têm sido executadas, em sua maior parte, por meio de projetos organizacionais (Bouer \& Carvalho, 2005). O uso da análise RBV-VRIO mostrou ser um poderoso instrumento de identificação e avaliação dos recursos e capacidades internos das empresas, para que uma vez identificados esses recursos, possam ser desenvolvidos ao ponto da competência, podendo contribuir para o alcance da vantagem competitiva sustentável.

As cinco características de maturidade em GP comuns às empresas pesquisadas apresentam um alinhamento com a teoria da internacionalização e ao processo desenvolvido pelos três casos: (a) Definição 
de Objetivos e Estratégias: durante o processo de internacionalização as empresas definem, além do mercado-alvo, os objetivos que serão perseguidos e as ações que sustentam a estratégia de se internacionalizar; (b) Gerenciamento da Mudança: a gestão da mudança observa também alterações em regas e acordos comerciais, assim como em comportamento e na análise dos impactos da cultura do mercado-alvo; (c) Gerenciamento de Riscos: a maior preocupação das empresas estudadas é com risco de alterações de legislação no mercado-alvo que possa inviabilizar a internacionalização, ou seja, na segurança jurídica da operação; (d) Envolvimento de Stakeholders: por se tratar de uma decisão estratégica da empresa, compromete toda sua estrutura e recursos, as partes interessadas devem ser envolvidas em todo o processo de internacionalização; (e) Sustentabilidade do Projeto: está relacionada à sustentação da empresa a partir da sua internacionalização em mercados internacionais e o quanto essa estratégia garante a sobrevivência ao longo do tempo.

Assim, percebe-se que existe uma convergência entre as características identificadas com as decisões cruciais das empresas durante o processo de internacionalização, o que permite sugerir que a empresa que pretende acessar mercados internacionais deve considerar as características da GP que contribuem com maior intensidade para internacionalização e consequentemente para uma vantagem competitiva sustentável durante esse processo.

O estudo traz como contribuição para prática e para as empresas brasileiras que pretender expandir seus negócios internacionalmente que as práticas maduras da GP podem ser consideradas recursos estratégicos para internacionalização de forma sustentável. Embora os resultados demonstrem que a maturidade da GP no nível 2 (processos comuns) não confere vantagem competitiva sustentável para empresa (características de escopo, custo e prazo são práticas fundamentais para excelência em GP), o estudo identificou as 5 características da gestão madura de projetos para implicação competitiva da empresa, categorizando segundo RBV-VRIO.

Algumas limitações deste estudo foram: (a) não investigação mais profunda da gestão dos projetos nas empresas, como a tipologia, número de membros na equipe, número de projetos, entre outras questões; (b) número reduzido de respondentes participantes, tanto na etapa de investigação da maturidade em GP, como na etapa de investigação da internacionalização e RBV-VRIO; (c) um único entrevistado para refletir a contribuição da GP para internacionalização da empresa sob a ótica do RBV, representando a visão da empresa.

Estudos futuros podem ser desenvolvidos explorando o uso das 5 características da maturidade em GP em empresas internacionalizadas, podendo atribuir outras análises na vantagem competitiva das empresas que as possuem.

Conclui-se que a análise das contribuições da maturidade na GP para internacionalização considerando a perspectiva da RBV mostrou-se adequada e válida pelos resultados obtidos. As práticas da maturidade na GP, quando apropriadas pelas empresas, contribuem para a internacionalização das empresas para entregar projetos dentro de prazos e orçamentos préestabelecidos, para o cumprimento de requisitos de fornecimento como qualidade e certificação, incluindo ainda no auxílio da avaliação de escopo, na gestão de riscos, e outros. A maturidade na GP contribui ainda para o alinhamento estratégico, das formas de entrada nos mercados e no relacionamento com os stakeholders das empresas analisadas, imprimindo uma vantagem competitiva frente aos concorrentes.

\section{REFERÊNCIAS}

- Almor, T., Hashai, N. \& Hirsch, S. (2006). The product cycle revisited: Knowledge intensity and firm internationalization. Management International Review, 46, 507-528.

- Andersen, O. (1993). On the Internationalization Process of Firms: A Critical Analysis. Journal of International Business Studies, Vol. 24 No. 2, pp. 209-231.

- Anderson, E. \& Gatignon, H. (1986). Modes of Foreign Entry: A Transaction Cost Analysis and Propositions. Journal of International Business Studies, Vol. 17, No. 3, pp.1-26.

- Andersson, S. (2000). The internationalization of the firm from an entrepreneurial perspective. International Studies of Management \& Organization. 30(1), 63- 93.

- Barney, J. B. (1991). Firm Resource and Sustained Competitive Advantage. Journal of Management, New York, v. 17 , n. 1, mar. pp. 99-121.

- Barney, J. B. (2001). The resource based "view" a useful perspective for strategic management research? Yes. Academy of Management Review, Vol. 26, No. 1, 41-56.

- Barney, J. B. \& Clark, D. N. (2007). Resource-Based Theory: Creating and Sustaining Competitive Advantage. New York: Oxford University Press. 
- Barretto, A. \& Rocha, A. (2003). A expansão das fronteiras: brasileiros no exterior. In ROCHA, A. (org) As novas fronteiras: a multinacionalização das empresas brasileiras. Rio de Janeiro: Mauad, 2003. (Coleção Estudos COPPEAD).

- Bouer, R. \& Carvalho, M. M. (2005). Metodologia Singular de Gestão de Projetos: Condição Suficiente Para a Maturidade em Gestão de Projetos? Revista Produção, v. 15, n. 3, p. 347-361, Set./Dez.

- Carvalho, M. M. \& Rabechini Jr, R. (2011) Fundamentos em Gestão de Projetos: Construindo Competências para Gerenciar Projetos. São Paulo: Editora Atlas, 3.ed.

- Cleland, D. I \& Ireland, L. R. (2008). Project Manager's Handbook: Applying Best Practices across Global Industries. New York: McGraw-Hill.

- Costa, C. P. \& Moura, H. P. (2009, p. 42). Modelos de Maturidade na Gestão de Projetos: Modelos Existentes e uma Análise Comparativa. Revista Engenharia de Software Magazine. n.18, pp.36-44.

- Dinsmore, P. C. (1999). Winning in business with enterprise project management. American Management Association, AMACOM, NY, USA.

- Dinsmore, P. C. \& Cabanis-Brewin, J. (2006). The AMA Handbook of Project Management. Second Edition. American Management Association, AMACOM, NY, USA.

- Douglas, S. P. \& Craig, C. S. (1995). Global Marketing Strategy. New York: McGraw-Hill.

- Dunning, J. H. (2001). The eclectic (OLI) paradigm of international production: past, present and future. International Journal of the Economics of Business, 8(2): 173-190.

- Eisenhardt, K. M. (1989). Building Theories from Case Study Research. The Academy of Management Review, 14(4), 532-550.

- Elango, B. \& Pattnaik, C. (2007), Building capabilities for international operations through networks: a study of Indian firms. In: Journal of International Business Studies, 38(4): 541-555.

- FDC, Fundação Dom Cabral, (2013). Ranking das Multinacionais Brasileiras. Disponível em: www.fdc.org.br.

- Floriani, D. E. (2010). O Grau de Internacionalização, as Competências e o Desempenho da PME Brasileira. [tese de doutorado]. Faculdade de Economia, Administração e Contabilidade - FEA/USP. São Paulo: USP.

- Floriani, D. E. \& Fleury, M. T. (2012). O Efeito do Grau de Internacionalização nas Competências Internacionais e no Desempenho Financeiro da PME Brasileira. Revista de Administração Contemporânea - RAC, Rio de Janeiro, v. 16, n. 3, art. 6, pp. 438-458.

- Goulart, L., Arruda, C. A. \& Brasil, H.V. (1994). A evolução da dinâmica de internacionalização. Revista Brasileira de Comércio Exterior, n. 41, p. 31-41, out-dez.

- Grant, R. M. (1991). The Resource-Based Theory of Competitive Advantage: Implications for Strategy Formulation. California Management Review, Vol. 33, Issue 3, pp. 114-135.
- Herkenhoff, D. A. (2010, p. 85). Análise Comparativa dos Modelos de Maturidade de Projetos: OPM3, CMMI, KERZNER e MMGP-Prado. Projeto Final de curso de MBA em Gerenciamento de Projetos. UFF: Niterói.

- Hatch, M. J. (1997) Organization Theory: Modern, SymbolicInterpretive and Postmodern Perspectives. Oxford: Oxford University Press.

- Hitt, M. A, Hoskissom, R. E \& Kim, H. (1997). International diversification: effects on innovation and firm performance in product-diversified firms. Academy of Management Journal. v. 40, n. 4, pp. 767-798.

- Hitt, M. A., Ireland, R. D. \& Hoskisson, R. E. (2011). Strategic Management - Competitiveness and Globalization, 9th ed. ITO, Cincinnati, Ohio, USA.

- Hollensen, S. (2011). Global marketing: a decision-oriented approach. 5th ed. England: Pearson Education Limited.

- Johanson, J. \& Vahlne, J-E. (1977). The internationalization process of the firm: A model of knowledge development and increasing foreign market commitments. Journal of International Business Studies, v.8, n.1, pp. 23-32.

- Johanson, J. \& Vahlne, J-E. (1990). The Mechanism of Internationalization. International Marketing Review, 7(4): 11-24.

- Johanson, J. \& Wiedersheim-Paul F. (1975), The Internationalization of the Firm - Four Swedish Cases, Journal of Management Studies, 12: 305-322.

- Kerzner, H. (2006). Gestão de Projetos: As Melhores Práticas. 2.ed. Porto Alegre: Bookman.

- Kerzner, H. (2009). Project management: a system approach to planning, scheduling, and controlling. 10. Edition. New York: John Wiley \& Sons.

- Kerzner, H. (2011). Gerenciamento de Projetos: Uma Abordagem Sistêmica para Planejamento, Programação e Controle. 10.ed. São Paulo: Blucher.

- Kraus, P. G. (2006). O Processo de Internacionalização das Empresas: O Caso Brasileiro. Revista de Negócios, Blumenau, v. 11, n. 2, p.25-47, Abril-Junho.

- Levine, H. A. (2002): Practical project management: tips, tactics, and tools. New York: John Wiley \& Sons, Inc.

- Lientz, B. P. \& Rea, K. P. (2003). International Project Management. Academic Press: Elsevier Science.

- Lopes, Daniel. (2009). Critérios de Avaliação de Desempenho de Gerenciamento de Projetos: Uma Abordagem de Estudo de Casos. Dissertação (Mestrado em Engenharia), São Paulo: Escola Politécnica / USP.

- Madeira, A. B. \& Silveira, J. A. G. (2013). Internacionalização de Empresas: Teorias e Aplicações. São Paulo: Saint Paul Editora.

- Madhok, A. (1997): Cost, value and foreign market entry mode: the transaction and the firm, in Strategic Management Journal, Vol.18, p.39-61

- Moreira, A. C. (2009). The Evolution of Internationalization: Towards a New Theory? Economia Global e Gestão, Vol. 14, No. 1, pp. 41-61. 
- Muriithi, N. \& Crawford, L. (2003). Approaches to project management in Africa: Implications for international development projects. International journal of project management. 21.05: Vol. 21. pp. 309-319.

- Penrose, E. T. (2006). A teoria de crescimento da firma. 3o ed. Tradução: Tamás Szmrecsányi. Campinas, SP: Editora da Unicamp.

- Peteraf, M. A. (1993). The Cornerstones of Competitive Advantage: A Resource-Based View. Strategic Management Journal, Vol. 14, No. 3. (Mar., 1993), pp. 179-191.

- PMI, Project Management Institute. (2008). A Guide to the Project Management Body of Knowledge (PMBOK Guide). 4 rd ed. Newtown Square, USA: PMI.

- Porter, M. E. (1993). A Vantagem Competitiva das Nações. Rio de Janeiro: Campus.

- Porter, M. E. (1996). "What is Strategy?", Harvard Business Review, November-December: 61-78.

- Prado, D. S. (2004). Gerenciamento de Programas e Projetos nas Organizações, 3.ed. Nova Lima: INDG-TECs, pp.85-100.

- Rabechini Jr. R. (2005). Competência e maturidade em gestão de projetos. 1.ed. São Paulo: Anna Blume,

- Root, F. R. (1987). Entry strategies for international markets. Lexington: Lexington Books.

- Sbragia, R., Rodrigues, I., Piscopo, M. \& Moreira, N. V. A. (2009) Gerenciamento de Projetos: Avanços e Tendências na Pesquisa Acadêmica. Revista Mundo PM (Curitiba), v. 5, pp. 52-58.
- Shenhar, A. J. (2004). Strategic Project Leadership ((R)) Toward a strategic approach to project management. Stevens Institute of Technology, Hoboken, USA. 34(5): 569578.

- Shenhar, A. J. \& Dvir, D. (2010). Reinventando Gerenciamento de Projetos: A abordagem Diamante ao Crescimento e Inovação Bem-sucedidos. São Paulo: M Books.

- Stanleigh, M. (2007, 30 August). Process management vs project management. Retrieved 17-03-2014, from: http://www.improvementandinnovation.com.

- Vernon, R. (1966). International investment and international trade in the product cycle. Quarterly Journal of Economics, 80 (2): 190-207.

- Vianna, N. W. H., Piscopo, M. R., \& Ryngelblum, A. (2013). Internacionalização da pequena e média empresa brasileira: o caso da indústria de máquinas-ferramenta. BASE - Revista de Administração e Contabilidade da UNISINOS, 10(3), pp. 210-223.

- Webster Jr, F. M., \& Knutson, J. (2006). What is Project management? Project management concepts and methodologies. In: P. Dinsmore, \& J. Cabanis-Brewin (Eds.), The AMA Handbook of project management (pp. 1 - 10). 2 Ed. Nova Yorque, NY: Amacom.

- Wernerfelt, B. (1984). A resource-based view of the firm. Strategic Management Journal, v.5, n.2, pp. 171-180, AprJun.

\section{Sobre os autores}

- André Luiz Spinelli Schelini possui Mestrado em Administração pela Universidade Nove de Julho, UNINOVE/SP, pós-graduado em Administração Pública, Gestão de Comunicação e Marketing Institucional e, ainda, docência do Ensino Superior pela Universidade Castelo Branco (Rio de Janeiro/RJ) E-mail: schelini@gmail.com

- Cristina Dai Prá Martens possui Mestrado e Doutorado em Administração pelo PPGA/EA/UFRGS); Pósdoutorado no CERAG/UPMF-Université Pierre Mendès-France. Professora do PPGA e MPA-GP UNINOVE.E-mail: cristinadm@uninove.br

- Marcos Roberto Piscopo - Mestrado e Doutorado em Administração pela FEA-USP, com estágio Doutoral na Universidade Bocconi, em Milão, Itália. Professor do MPA-GP UNINOVE. Ad memoriam. 


\title{
The project management as competitive advantage for internationalization Brazilian companies
}

\author{
André Luiz Spinelli Schelini, Cristina Dai Prá Martens, and Marcos Roberto Piscopo
}

Universidade Nove de Julho - UNINOVE, São Paulo, SP,Brazil

\begin{tabular}{l}
\hline ARTICLE DETAILS \\
\hline Article history: \\
Received: April $16^{\text {th }}, 2017$ \\
Accepted: June $26^{\text {th }}, 2017$ \\
Available online: December $12^{\text {th }}, 2017$ \\
Double Blind Review System \\
Scientific Editor \\
Ilan Avrichir
\end{tabular}

\section{Keywords:}

Project Management Maturity

Internationalization of companies Resource-

Based View (RBV)

Brazilian companies

Sustainable Competitive Advantage

\begin{abstract}
This study focuses on three theoretical axes: project management (PM), internationalization of companies and the resource-based view (RBV). It aimed to analyze if, and in what way, PM maturity contributes to the international strategy of Brazilian companies from the RBV perspective. The research strategy adopted in three Brazilian companies constituted a multiple case study with a descriptive exploratory approach through intercase and intracase analysis. As an unprecedented result, the study identified five characteristics of mature project management: project sustainability, stakeholder engagement, goal setting and strategy, change management, and risk management. Furthermore, all of these contributed to the internationalization of the companies in question, with an impact on competitive advantage, considering the RBV-VRIO analysis. Thus, the study contributes to the field by aiding companies that are beginning or intend to begin internationalization processes, enabling them to obtain better results through PM. A limitation of the study is the small number of cases investigated, which may be viewed as an opportunity for conducting further studies.
\end{abstract}

(C) 2017 Internext | ESPM. All rights reserved!

To cite this article:

Schelini, A. L. S., Martens, C. D. P., \& Piscopo, M. R. (2017). Project management as a competitive advantage for the internationalization of Brazilian companies. Internext - Revista Eletrônica de Negócios Internacionais, 12 (3), 01-15. DOI: http://dx.doi.org/10.18568/1980-4865.1231-15

To access this article: $h t t p: / / d x$. doi.org/10.18568/1980-4865.1231-15 